\title{
Solitary wave calculations for erosion strength
}

\author{
H. Barth \& E. R. Hilf \\ Fachbereich Physik, Carl von Ossietzky Universität Oldenburg; \\ Carl-von-Ossietzky-Str. 9-11, D-26111 Oldenburg, Germany
}

ABSTRACT: For the calculation of steep water waves, a Lagrangian method is presented. This makes it easier to derive and understand the complicated non-linear structure of the equations of motion (EOM), for the behaviour of water surfaces. In addition, this formulation offers the possibility to model an EOM which describes water waves with deference to variation in depth.

\section{INTRODUCTION}

Water waves with larger amplitudes, both in deep and in shallow water, have relatively different properties in contrast to the well-known low-amplitude sinoidal waves. The properties of steep water waves, travelling into shallower regions of the shore, may be of interest for marine biology due to their impact on, for example, water flow turbulence, sea bottom erosion, chemical exchange with the atmosphere or momentum and energy transfer. Presented in this paper is a way of calculating their properties.

The behaviour of water waves could be calculated from hydrodynamics if the exact way of linearization of the basic equations were known. This, however, is the problem. Thus, various model assumptions have been proposed and respective equations of motion set up. These have, however, a complicated mathematical structure. This made it difficult to understand the basic properties in terms of the equation of motion (EOM). In addition, most solutions have been gained by linearizing (and thus simplifying) the EOM. It turns out, however, that the larger amplitude waves have non-linear properties, which are inadvertantly not being considered.

We propose to start the derivation of an EOM by a Lagrangian function of the transverse surface field. The design of Lagrangians is known to be suitable for the case of complicated and coupled motions in Abstract Quantum Field Theory. The advantage is that each field enters with additive kinetic and potential terms. The coupling between different fields, as well as the non-linear selfcoupling, enter by relatively simple additive terms. This greatly helps the design of a Lagrangian which is suited to a given set of phenomena.

As an example, assume a water basin with the surface elevation, $\eta(z)$. Then the Lagrangian has to have a kinetic term, $\frac{1}{(2 \mathrm{~m})}(\partial \eta)^{2}$, with the moment of inertia, $\mathrm{m}$, and a potential (or mass term), $(\eta)^{2}$, both of which serve an EOM for the determined free or linear waves. If this surface Lagrangian is modelled to have in addition higher order terms, such as $(\eta)^{\mathrm{n}}$, where $\mathrm{n}=3,4,5 \ldots$, these turn out to be responsible for self-coupling non-linear effects. One of which is the well studied solitary wave or soliton. 
Because of the higher powers of the Lagrangian terms, one sees immediately that these solitons can occur only if the amplitudes $\eta$ are sufficiently high. Otherwise, an $\eta^{3}$ term would be small against the terms of the free waves and, thus, would not contribute to the resultant EOM and its final solution. Sometimes the soliton regime of the EOM is called "weak non-linear", because one still has solution waves, however with different properties.

In the case of more than one field, as in the mentioned surface waves and now, in addition, a longitudinal sound or other longitudinal disturbance $\chi_{1}$ the Lagrangian method has the advantage of easily coping with it, by just introducing the respective kinetic and potential term for the new field plus a coupling term between these two fields, by adding a product of the type $\eta^{2} \chi$. This procedure has proven its validity in many fields of coupled motions, and could even be applied to the coupling of ocean sea quakes with the ocean surface waves, known as "Tsunami".

In this paper we design and apply the Lagrangian method to the following cases: (1) ocean waves in deep water being coupled to themselves and (2) surface waves in shallow water being coupled to the depths of the water column. We will set up the respective Lagrangian, derive the resulting EOM and discuss the properties of the solutions in comparison to linear waves.

\section{PROPERTIES OF SOLITARY WAVES}

Conventional low amplitude s in o id al water surface waves are known to have the following properties:

- The amplitude is independent of its wavelength;

- The velocity depends on the wavelength (shorter waves travel more slowly). Wave packages disperse, disassemble with time.

In contrast, solitons demonstrate the following properties:

- The amplitude depends on the velocity (or other parameters) of the wave and vice versa;

- No dispersion: a solitary wave is stable in its form with time.

Thus, a soliton is a local, stable but propagating object.

We demonstrate the advantage of the Lagrangian Method using applications for both shallow- and deep-water waves.

\section{SHALLOW WATER WAVES}

\section{The equation of motion}

The equation of motion for shallow-water waves has been extensively discussed in the literature (Whitham, 1974; Ablowitz \& Segur, 1981). Their complicated, intricate and nonlinear structure is exemplified by the well studied Korteweg-de Vries equation. This is a differential equation for the wave amplitude, $\eta$, as a function of the water depth, $h_{0}$, travelling along $x$ with time, $t$. The constant, $c_{o r}$ gives the coupling between the depth and $\eta$; the strength of which is governed by the gravitational acceleration $g, c_{0}=\sqrt{g h_{0}}$. This equation holds only for constant water depth, $h_{o}=$ const. 


$$
\frac{\partial \eta}{\partial t}-c_{0}\left(1+\frac{3}{2} \frac{\eta}{h_{0}}\right) \frac{\partial \eta}{\partial x}+\frac{1}{6} c_{0} h_{0}^{2} \frac{\partial^{3} \eta}{\partial x^{3}}=0
$$

The solutions are:

$$
\eta=\eta_{0} \operatorname{sech}^{2}\left\{\left(\frac{3 \eta_{0}}{4 h_{0}^{3}}\right)^{1 / 2}(x-v t)\right\}
$$

These Korteweg-deVries solutions refer to one of the oldest cases of a soliton, with all its properties as mentioned above. Specifically, the velocity is given by:

$$
\mathrm{v}=c_{0}\left(1+\frac{1}{2} \frac{\eta_{0}}{h_{0}}\right)
$$

That is, the higher the soliton, the faster it is.

Although the KdV equation does give interesting soliton solutions, the EOM, at least to us, is by no means vivid. Thus, no feeling of confidence may be developed to design a respective EOM for more complicated cases, such as rapidly changing depths.

\section{The Lagrangian}

For a linear sinoidal wave, the Lagrangian, for the surface amplitude, $\eta$, has to have a kinetic and a "free" or so called "mass" term. It is:

$$
L=\frac{1}{2}\left(\frac{\partial \eta}{\partial x}\right)^{2}+\alpha_{1} \eta^{2}
$$

The simplest non-trivial Lagrangian needs to have at least one additional term. It is an equation with a cubic term in $\eta$. This is:

$$
L=\frac{1}{2}\left(\frac{\partial \eta}{\partial \mathrm{x}}\right)^{2}+\alpha_{2} \eta^{2}+\beta_{2} \eta^{3}
$$

This leads, by the proper derivatives, directly to the non-linear Korteweg-deVries equation of motion. In contrast to its intricate mathematical structure, the Lagrangian consists only of additive terms, where the cubic in $\eta$ is known to be responsible for soliton wave type solutions.

\section{DEEP-WATER WAVES}

\section{The Lagrangian}

Thus, we apply this method of constructing a Lagrangian and from there we calculate the respective equation of motion to the non-linear Schrödinger equation as well. The repective Lagrangian is:

$$
L=\frac{1}{2}\left(\frac{\partial \eta}{\partial x}\right)^{2}+\alpha_{3} \eta^{2}+\beta_{3} \eta^{4}
$$

The biquadratic term is known to be responsible for a "self-coupling" of waves. 


\section{Generalization}

While the Korteweg-deVries case is for coupling to the shallow water at the seabottom, the non-linear Schrödinger is valid for deep-water self-coupling of surface waves.

The generalization for steep-water waves for any water depths including shallow water may be impossible to find, starting directly from the equations of motion. For the Lagrangian method, it is extremely easy. We just add the two non-linear terms to the following formula:

$$
L=\frac{1}{2}\left(\frac{\partial \eta}{\partial x}\right)^{2}+\alpha \eta^{2}+\beta \eta^{3}+\gamma \eta^{4}
$$

The coefficients now need to be desgned so that the two limiting cases are included.

$\alpha$ has to go continuously from $\alpha_{2}$ to $\alpha_{3}$ as a function of depth and for small amplitudes to $\alpha_{1}$. From first principles they should be roughly equal.

$\beta$ should be set up to depend on the inverse of the depth.

Finally, $\gamma$ has to be zero for shallow water. A suitable placement may thus be $\left.\mathrm{a} /\left(1+\exp (1)-h_{o}\right) b\right)$ with a coupling strength $a$ and a coupling "width" $b$.

At the moment, we evaluate these parameters to be optimally applicable to seawater waves travelling from deep to shallow water.

\section{EXAMPLES}

In Figures 1,2 and 3 we show some example of specific calculations. To get the EOM from the Langrangian, one needs to use the well-known Variational Principle and afterwards solve the differential equation (EOM).

A deep water soliton solution is shown in Figure 1. It is just a group of three waves, locally concentrated in space in both surface directions.

Travelling into shallower water, the front- and after-waves diminish to feed the main central wave. The wave length perpendicular to the travelling direction increases (Fig. 2).

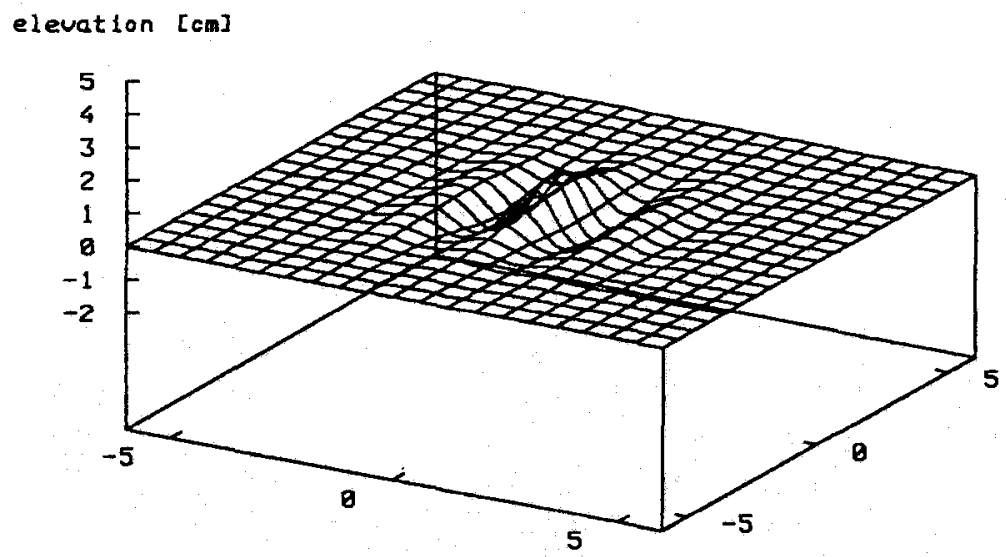

Fig. 1. Deep-water soliton 


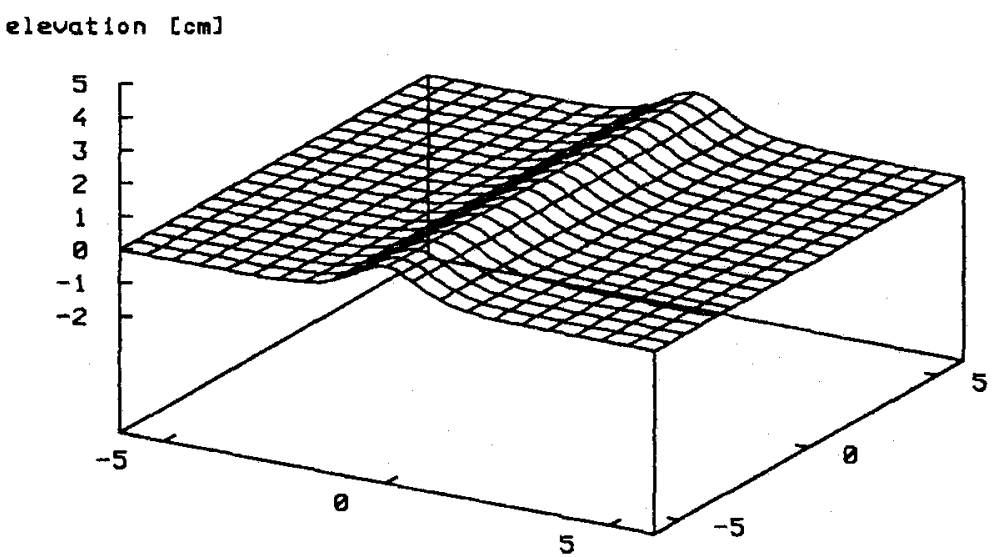

Fig. 2. Shallow-water soliton

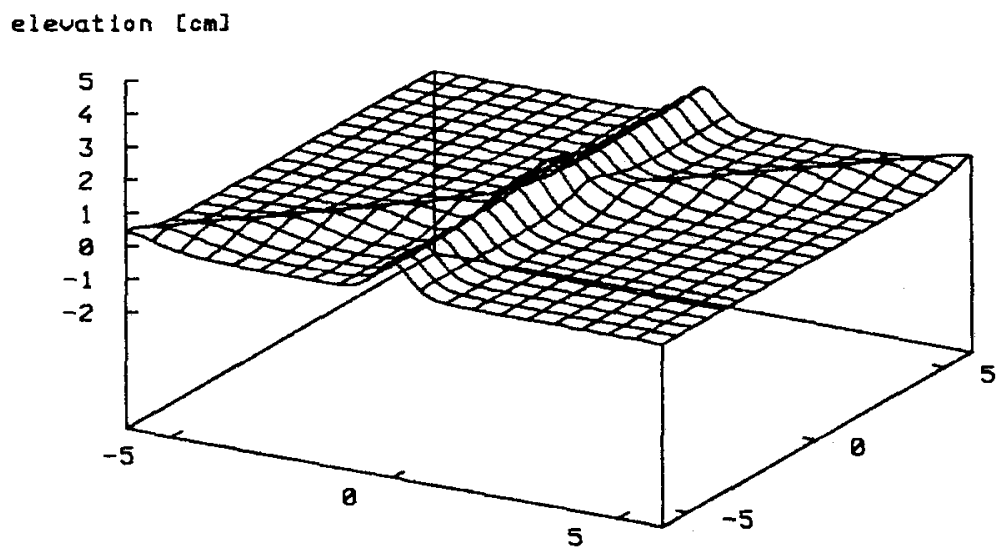

Fig. 3. Calculation for two crossing shallow-water solitons

In Figure 3 we give a special example for the case of two crossing shallow water solitons. This can be compared to the photograph from Ablowitz \& Segur (1981) which exhibits this case (Fig. 4).

\section{SUMMARY}

A new method has been presented to design equations of motion for deep-water waves travelling in deep or shallow water. Their solutions give their properties, needed as input to marine biology scenarios, both close to the sea's surface and in shallow water. 


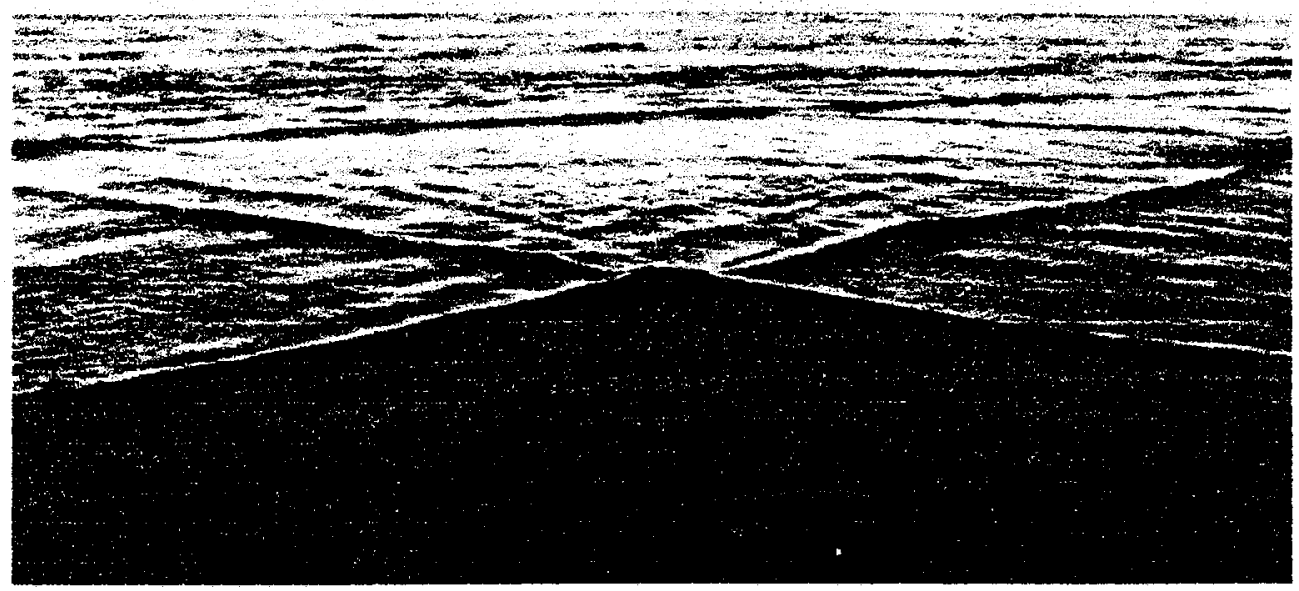

Fig. 4. Interaction of two shallow-water waves

Acknowledgements. Stimulating discussions with B. and J. Curdes, as well as with J. Barghorn, are acknowledged. For our own additional intuitive instruction and for object teaching of my colleagues, we enjoyed the excursion of the Sailing R. V. Isern Hinnerk from Rendsburg to Helgoland and from there to Jemgum/Ems in suitably rough weather conditions. We gratefully acknowledge the support of young students by the Biologische Anstalt Helgoland, and are indebted especially to Dr. A. Gebhardt for perfect organization.

\section{LITERATURE CITED}

Ablowitz, M. J. \& Segur, H., 1981. Solitons and the inverse scattering transform. Siam, Philadelphia, 425 pp.

Whitham, G. B., 1974. Linear and nonlinear waves. Wiley, New York, $636 \mathrm{pp}$. 